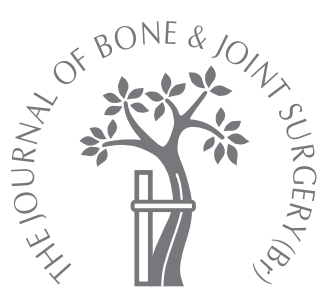

L. A. Moroz,
F. Launay,
M. S. Kocher,
P. O. Newton,
S. L. Frick,
P. D. Sponseller,
J. M. Flynn

From The Children's

Hospital of

Philadelphia, Timone

Children's Hospital,

Marseille, Children's

Hospital, Boston,

Children's Hospital,

San Diego, Carolinas

Medical Center,

Charlotte, Johns

Hopkins Hospital,

Baltimore

- L. A. Moroz, BA, Medical Studen J. M. Flynn, MD, Orthopaedic

Surgeon

Department of Orthopaedic Surgery Children's Hospital of Philadelphia, 2nd Floor Wood Building, 34th Street \& Civic Center Boulevard, Philadelphia Pennsylvania 19104-4399, USA.

E. Launay, MD, Orthopaedic

Surgeon

Department of Orthopaedic Surgery Timone Children's Hospital, 264 St-

Pierre Street, 13005 Marseille, France

Pierre Street, 13005 Marseille, France.

= M. S. Kocher, MPH, MD,

Orthopaedic Surgeon

Department of Orthopaedic Surgery

Children's Hospital, Harvard Medic

School, 300 Longwood Avenue,

Boston, Massachussetts 02115, USA.

- P. O. Newton, MD, Orthopaedic

Surgeon

Department of Orthopaedic Surgery

Children's Hospital San Diego, 3030

Children's Way, San Diego, California

92123, USA.

E. S. L. Frick, MD, Orthopaedic

Surgeon

Department of Orthopaedic Surgery Carolinas Medical Center, 1000 Blythe Boulevard, Charlotte, North Carolina 28232, USA.

= P. D. Sponseller, MD, Orthopaedic

Surgeon

Department of Orthopaedic Surgery

Johns Hopkins Hospital, 601 North

Carolina Street, Baltimore, Maryland

Correspondence should be sent to $\mathrm{Dr}$

J. M. Flynn

e-mail: flynnj@email.CHOP.edu

(๑2006 British Editorial Society of

Bone and Joint Surgery

doi:10.1302/0301-620X.88B10

$17517 \$ 2.00$

$J$ Bone Joint Surg $[B r]$

2006;88-B:1361-6.

Received 7 December 2005: Accepted after revision 31 May 2006

\title{
Titanium elastic nailing of fractures of the femur in children
}

\author{
PREDICTORS OF COMPLICATIONS AND POOR OUTCOME
}

\begin{abstract}
Between 1996 and 2003 six institutions in the United States and France contributed a consecutive series of 234 fractures of the femur in 229 children which were treated by titanium elastic nailing. Minor or major complications occurred in $\mathbf{8 0}$ fractures. Full information was available concerning 230 fractures, of which the outcome was excellent in $150(65 \%)$, satisfactory in $57(25 \%)$, and poor in $23(10 \%)$. Poor outcomes were due to leglength discrepancy in five fractures, unacceptable angulation in 17, and failure of fixation in one. There was a statistically significant relationship $(p=0.003)$ between age and outcome, and the odds ratio for poor outcome was $\mathbf{3 . 8 6}$ for children aged 11 years and older compared with those below this age. The difference between the weight of children with a poor outcome and those with an excellent or satisfactory outcome was statistically significant (54 $\mathrm{kg}$ vs $39 \mathrm{~kg} ; \mathrm{p}=\mathbf{0 . 0 0 3}$ ). A poor outcome was five times more likely in children who weighed more than $49 \mathrm{~kg}$.
\end{abstract}

Fractures of the diaphysis of the femur are common in children. Various methods of treatment can be used successfully, depending on the age of the child and the type of fracture. Immediate application of a spica cast, or traction followed by a cast, remains the standard management for most of these fractures in children younger than six years of age. ${ }^{1-4}$ In skeletally-mature adolescents, antegrade solid intramedullary nailing is usually employed. ${ }^{5,6}$

Titanium elastic nailing has been commonly used for treating fractures of the femur in children in Europe for two decades. ${ }^{7-9}$ It has more recently become a widely-accepted option for orthopaedic surgeons in the United States, where similar fractures had been managed by external fixation or traction and spica casting.

During the last ten years titanium elastic nails have become the most widely-used treatment for fractures of the diaphysis of the femur in children of school age in the United States. ${ }^{10-12}$ A number of previous studies have focused on the safety and efficacy of this method in patients between the ages of six and 16 years. $4,7,13,14$ Our study attempts to refine the indications for the use of these nails and identifies factors which might predict a poor outcome.

\section{Patients and Methods}

After obtaining approval from the respective Institutional Review Board, one attending surgeon from each of the six level I trauma centres identified a consecutive series of fractures of the femur treated with titanium elastic nails between 1996 and 2003. All patients between three and 18 years of age with a fracture of the diaphysis who had been treated with two intramedullary nails were included. All had been followed up until the fracture had healed or the nails removed. Pathological fractures were excluded. A data sheet was completed for each patient using information from the medical records, and by reviewing imaging studies. The age, weight, mechanism of injury, associated injuries, intra-operative problems, and operative complications were recorded. The pre-operative radiographs were evaluated to determine the location and pattern of the fracture.

We assessed a total of 229 children with 234 fractures of the diaphysis of the femur. The centres (The Children's Hospital of Philadelphia, Timone Children's Hospital, Children's Hospital Boston, Children's Hospital San Diego, Carolinas Medical Center and Johns Hopkins Hospital) contributed $43.6 \%$ of the fractures (102), $12.8 \% \quad(30), 12.4 \% \quad(29)$, $11.5 \%$ (27), $10.3 \%$ (24), and $9.4 \%$ (22), respectively. There were 171 males $(74.7 \%)$ and 58 females $(25.3 \%)$. The mean age at the time of fracture was 10.3 years ( 3 to 18 ) (Fig. 1). The mean weight was $40 \mathrm{~kg}$ (17 to 96). The most common mechanism of injury was a motor vehicle accident as a passenger or a 


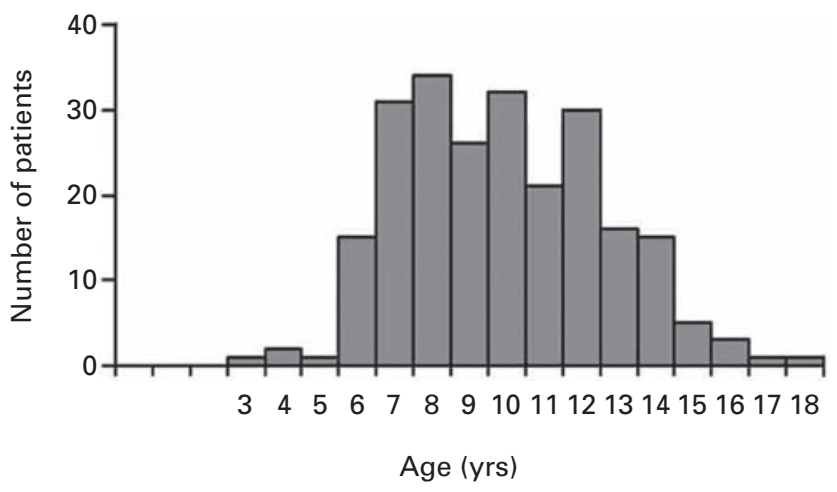

Fig. 1

Distribution of ages of patients at the time of treatment.

pedestrian in 136 fractures ( $58.1 \%$ ), followed by a sporting injury in $46(19.6 \%)$, and a fall from a height in 43 $(18.4 \%)$. Multiple injuries were present in 66 patients $(28.8 \%)$ including 19 with head injuries, 17 with visceral injury and 51 with associated fractures.

The standard technique for use of titanium elastic nails has been described elsewhere. ${ }^{15}$ The pattern of immobilisation after operation varied widely among the 39 surgeons who treated these fractures.

The information collected at visits after operation included the range of movement in the hip and knee, rotational alignment, time to weight bearing and nail removal, signs of irritation or local infection from the tip of the nail, and signs of deep infection and post-operative complica- tions. The leg length was evaluated clinically using either tape measurement or a block test. A scanogram was performed at the discretion of the physician when there was clinical evidence of discrepancy in leg-length.

Post-operative radiological evaluation included an assessment of the position of the nail, frontal and sagittal alignment, loss of reduction, callus formation, disturbance of trochanteric growth, osteonecrosis of the femoral head and femoral length. Varus and valgus angulation was measured from the anatomical axis. Flexion and extension was measured directly with the understanding that the normal bow of the femur contributes to anterior angulation of fractures of the midshaft by a few degrees. Circumferential callus formation, the visibility of the fracture line, and leglength discrepancy and malalignment were recorded at each post-operative visit in order to monitor remodelling and detect delayed union or deformity.

The senior author (JMF) compiled the data from all the centres in a single database. Complications were classified as minor when they resolved without additional surgery or did not result in long-term morbidity, and major when further operation was required or long-term morbidity ensued (Table I). We classified the final outcome as excellent when there was anatomical or near anatomical alignment with no peri-operative problems, satisfactory when there was acceptable alignment and leg length with resolution of perioperative problems, and poor in the presence of unacceptable alignment or leg length with unresolved peri-operative problems. Age-based criteria for assessing alignment were derived from standard guidelines. ${ }^{16}$ We chose stringent criteria so that all problems would be identified, recognising that even children with the poorest outcomes would subse-

Table I. Complications

\begin{tabular}{|c|c|}
\hline & $\begin{array}{l}\text { Number of fractures } \\
\text { (\% of } 234 \text { total) }\end{array}$ \\
\hline \multicolumn{2}{|l|}{ Minor } \\
\hline Pain at the site of nail insertion & $38(16.2)$ \\
\hline Minor angulation at final follow-up & $10(4.3)$ \\
\hline Minor leg-length discrepancy at final follow-up & $10(4.3)$ \\
\hline Inflammatory reaction due to nails & $5(2.1)$ \\
\hline Superficial infection at site of nail insertion & $4(1.7)$ \\
\hline $\begin{array}{l}\text { Delayed union (no sign of any callus after six wks or no } \\
\text { circumferential callus after six mths) }\end{array}$ & $2(0.9)$ \\
\hline $\begin{array}{l}\text { Loss of knee movement (worse than } 10^{\circ} \text { to } 110^{\circ} \text { ) more than two } \\
\text { mths after removal }\end{array}$ & $2(0.9)$ \\
\hline Total & $71(30.4)$ \\
\hline \multicolumn{2}{|l|}{ Major } \\
\hline Angulation exceeding the guidelines at the final follow-up* & $18(7.7)$ \\
\hline $\begin{array}{l}\text { Loss of reduction (between fixation and removal) requiring } \\
\text { new reduction or surgery }\end{array}$ & $9(3.8)$ \\
\hline Surgery to revise nail placement (e.g. nail trimming) & $6(2.6)$ \\
\hline Leg-length discrepancy exceeding guidelines at final follow-up & $5(2.1)$ \\
\hline Deep infection & $2(0.9)$ \\
\hline Haematoma requiring surgery & $1(0.4)$ \\
\hline Neurological damage after nailing & $0(0)$ \\
\hline Delayed or nonunion leading to revision & $0(0)$ \\
\hline Total & $41(17.5)$ \\
\hline
\end{tabular}


quently have normal or near normal function. The criteria for assessing leg-length discrepancy were based on the potential for further growth and whether further surgery would be considered.

Of the 234 fractures, no variable was missing for more than $8 \%$ of the possible data points. In four fractures in four patients, the radiological and clinical information was insufficient to determine outcome and they were excluded from the analysis. When the outcomes were analysed, patients missing the data point for the variable of interest were excluded from that analysis. A total of 230 fractures had sufficient data to classify outcome.

Statistical methods. Univariate analysis was used to test for correlations between each of the variables and the outcomes. Initially, we performed frequency analysis with chisquared statistics. A Fisher's exact test was also conducted because the total was less than five in many categories, making chi-squared statistics unreliable. Based on the results of the univariate analysis, we submitted our data for multivariate analysis to control for confounding variables and to determine if we could predict outcome. We used a stepwise method for variable selection in logistic regression analysis. Differences with a p-value of 0.05 or less were considered statistically significant. We used a Receiver Operating Characteristic (ROC) curve to analyse the relationship between weight and outcome. In order to detect groups at higher risk for poor outcome, the range of data was divided by 100 to be tested as a sequence of cut-off points. At each of these cut-off points a categorical variable was generated to create a set of binaries. By fitting this variable into a logistical model to predict outcome (poor, or excellent and satisfactory), the area under the ROC curve (the c-statistic) was obtained for this cut-off point, thus providing a measure of discrimination.

\section{Results}

The fracture occurred in the upper third of the femoral shaft in 33 cases $(14.1 \%)$, the middle third in $165(70.5 \%)$, and the lower third in $35(15 \%)$. The site of the fracture could not be determined in one case $(0.4 \%)$. There were 114 transverse $(48.7 \%), 47$ short oblique $(20 \%), 29$ spiral $(12.4 \%), 14$ butterfly $(6 \%)$, and 28 comminuted fractures $(12 \%)$. The fracture pattern could not be determined in two cases $(0.9 \%)$.

A total of 39 surgeons performed the operations. All of the fractures were treated with two titanium elastic nails. Nails of the same diameter were used in $96 \%$ of the fractures for which the size of the nail was recorded (215 of 224 fractures) and $83.5 \%$ (374 of 448 nails) were of either $3.5 \mathrm{~mm}$ or $4.0 \mathrm{~mm}$ in diameter. Based on an assessment of available post-operative radiographs, $82 \%$ (189 of 231) of the fractures had nails which formed a divergent $\mathrm{C}$ configuration; cortical contact by both nails was visible in $65 \%$ (148 of 228) and the nails crossed above and below the fracture site in $79 \%$ (180 of 228). The surgeon recommended post-operative immobilisation in a cast in 52 frac-

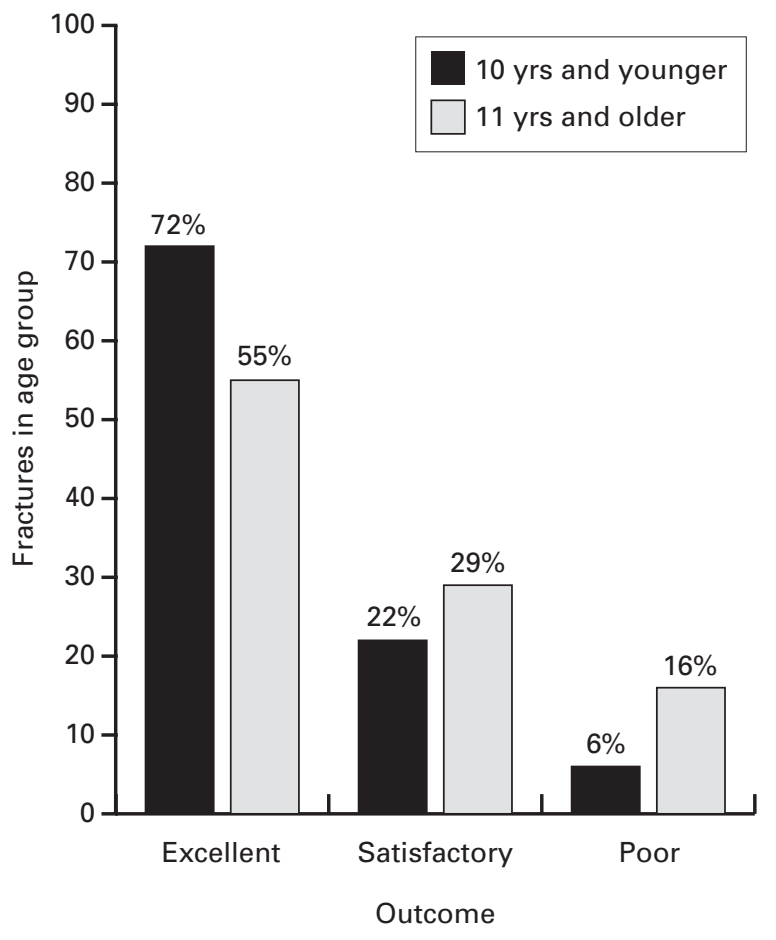

Fig. 2

The graph shows the proportion of excellent, satisfactory and poor outcomes for patients aged 10 years and younger compared with patients aged 11 years and older.

tures $(22.2 \%)$ and a knee immobiliser in 146 (62.4\%). Immobilisation was not employed for 33 fractures (14.1\%) and in three fractures $(1.3 \%)$, the method of post-operative immobilisation could not be determined from the available records.

Each patient was followed up until their fracture had healed or until the last follow-up visit. The mean length of follow-up was 14.2 months ( 2 to 71), and 212 of the 230 fractures for which an outcome classification could be determined were followed up for at least six months. The time to removal of the nails varied from 1.5 to 27 months (mean 6.7).

Complications. There were 112 complications during the post-operative follow-up in 80 fractures (Table I).

There was a strong association between proximal fractures and loss of reduction in children aged ten years and younger $(\mathrm{p}=0.046)$ and a strong association between distal fractures and loss of reduction in children aged 11 years and older $(\mathrm{p}=0.025)$. However, after adjusting for age, the strength of the association between the fracture site and loss of reduction $(p=0.17)$ was lost. There was no difference in the rate of malunion between fractures in the proximal, middle, or distal third of the femur for either the poor outcome $(\mathrm{p}=0.5453)$ or the satisfactory $(\mathrm{p}=0.6482)$ groups. In addition, there was no significant association between the clinical or radiological data and each type of complication. 


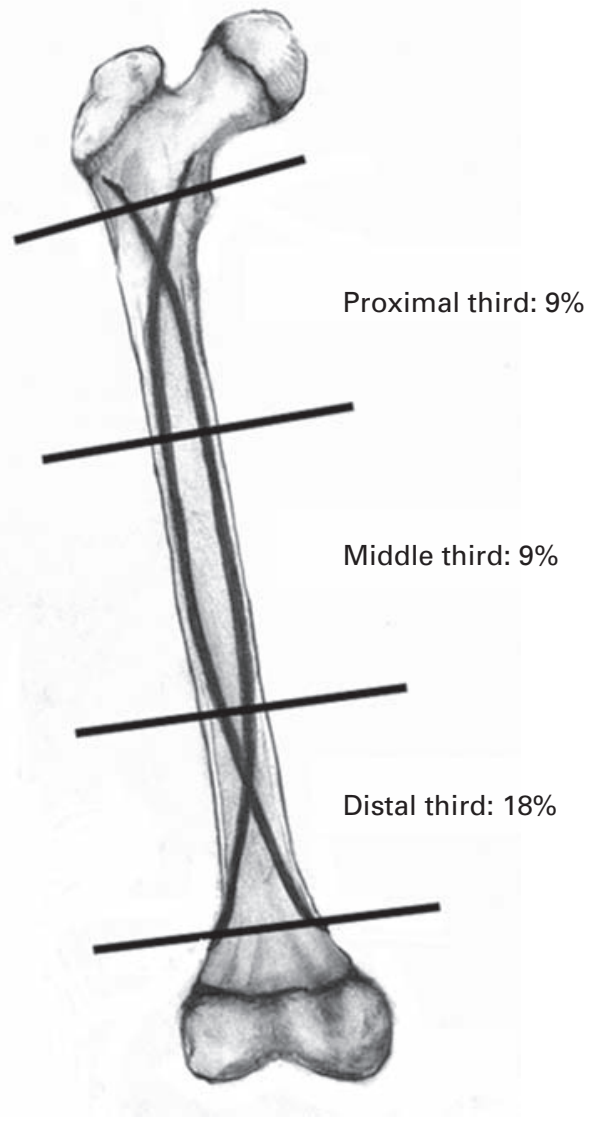

Fig. 3

Poor outcomes by location of the fracture.

Outcomes. The outcome was excellent in 150 fractures $(65 \%)$, satisfactory in $57(25 \%)$, and poor in $23(10 \%)$. We did not have enough information to classify four fractures which were excluded from the data analysis. With the numbers available, we could detect no statistical difference between the outcomes reported by the various centres $(\mathrm{p}=$ $0.375)$. In order to establish that differences in outcomes were not because of a learning curve of the surgeons as they adopted the use of titanium elastic nails, we compared the outcome of the first ten operations at each centre with the outcome of all their other cases. There was no statistical difference between the first ten procedures and the remain$\operatorname{der}(\mathrm{p}=0.298)$.

The final results were better in children aged ten years and younger than in those aged 11 years and above. In children aged ten years and younger, the results were excellent in 100 fractures $(71.9 \%)$, satisfactory in $31(22.3 \%)$, and poor in eight $(5.8 \%)$. In children aged 11 years and above, the results were excellent in $50(54.9 \%)$, satisfactory in 26 $(28.6 \%)$, and poor in $15(16.5 \%)$ (Fig. 2$)$. The outcome was poor in a higher percentage of fractures in the distal third, 6 of $33(18 \%)$, as compared with 14 of $164(9 \%)$ in the middle third and 3 of $33(9 \%)$ in the proximal third (Fig. 3), although the difference was not statistically significant.

The most significant association in the linear regression model was the age of the child $(<10$ years $v s>11$ years) and outcome $(p=0.003)$. There was no statistically significant difference between outcome with respect to the fracture characteristics, including pattern and site, or between the result with respect to the position of the nail following fixation. The divergent $\mathrm{C}$ configuration of the nails, cortical contact at the fracture site, or whether the nails crossed above and below the fracture did not influence the outcome. The mean time until removal of the nails was 6.4 months (1.8 to 13) in the poor group, 6.6 months (1.5 to 22 ) in the satisfactory group and 7 months (1.8 to 23) for the group with an excellent outcome. The mean time between fixation and full weight bearing was 10 weeks ( 6 to 18 ) for the poor outcome group, 10.2 weeks ( 3 to 28 ) for the satisfactory group and 9.1 weeks ( 3 to 32 ) for the excellent group. Neither the differences in the mean times between fixation and removal of the nails nor the times between fixation and full weight bearing were statistically significant. There was no statistically significant association within different age groups and adjusting for age did not yield significant results. Independently, however, age was a strong predictor of outcome: the odds ratio for poor outcome was 3.86 for children aged 11 years and older compared with children below this age. Since weight is associated with age, it did not add to the model.

Weights were recorded in 222 patients whose outcomes were classified. The mean weight was $39 \mathrm{~kg}$ (17 to 95.2) for the group including both excellent and satisfactory outcomes, and $54 \mathrm{~kg}$ (22.3 to 95.2) for the poor group. There was a significant difference between the variance of the two groups $(\mathrm{p}=0.01)$. A $t$-test using unequal variances was performed. There was a significant weight difference between the excellent and satisfactory outcome group and the poor outcome group $(p=0.003)$. In order to detect a weight group at higher risk for poor outcome, the range of weight data (17 to $96 \mathrm{~kg}$ ) was tested for cut-off points. Thus, at a cut-off point of $68.2 \mathrm{~kg}$, if a patient's weight is less than this the categorical weight is 'low', otherwise it is 'high'. By fitting this categorical weight variable into a logistic model to predict outcome (poor or excellent and satisfactory), c-statistics were obtained (area under the ROC curve) for this cut-off point. At a cut-off weight of $49 \mathrm{~kg}$ (c-statistic 0.68 ), a patient in the high weight group was approximately five times more likely to have a poor outcome. Because the cvalue was less than $0.75(0.68)$, the specificity was tested (Table II). Statistically, the weight cut-off point at $49.3 \mathrm{~kg}$ was not a sensitive test for detecting poor outcome $(78.5 \%$ specificity, $59 \%$ sensitivity).

\section{Discussion}

'New' devices or techniques generally tend to undergo a three-part analysis in the medical literature. The initial 
Table II. Test of sensitivity and specificity of weight at a cut off point of $49.3 \mathrm{kgs}$

\begin{tabular}{llc}
\hline & \multicolumn{2}{l}{ True outcome } \\
\cline { 2 - 3 } Predicted outcome & Poor & Excellent and satisfactory \\
\hline Poor & 13 & 43 \\
Excellent and satisfactory & 9 & 157 \\
\hline
\end{tabular}

reports are positive, but limited in scope and hampered by a learning curve and narrow indications. ${ }^{14}$ The device or technique is then compared with the optimum level of that in current use. In the case of titanium elastic nails, the results were shown to be better than either external fixation ${ }^{17}$ or spica casting. ${ }^{1}$ Following the publication of such studies, one risk is that surgeons widely adopt the technique and begin to stretch indications. Many surgeons noted a higher rate of problems when the technique was used improperly or when used in bigger, older and heavier children. ${ }^{10,18}$ This large, multicentre analysis has attempted to refine the indications and determine cases where a poor outcome becomes more likely.

The learning curve for implanting titanium elastic nails is not as steep as that of more technically challenging procedures. The device itself is simple to use and images can be obtained throughout the procedure to confirm proper alignment and fixation. Titanium elastic nailing has indeed been widely adopted: the centres from across the US and the centre in France that participated in this study had already made titanium elastic nails a standard treatment option by 1996. The surgeons had likewise reached proficiency with the operative technique, as is demonstrated by the outcomes, which are mostly excellent. There was no significant difference between the outcomes of cases performed earlier in our series and those of subsequent cases $(\mathrm{p}=0.298)$.

We collected a large, diverse group of children and adolescents whose femoral fracture had been treated with titanium elastic nails and then applied a rigorous statistical analysis to find relationships between complications, poor outcome and the many variables of demography and treatment. Earlier studies to evaluate complications associated with the technique ${ }^{10,14,17,18}$ have been of small size and from single centres. Although many studies have recommended titanium elastic nails as the treatment of choice in children aged between six and 12 years, the age of our patients ranged between three and 18 years. Patients older than 12 years comprised $17.5 \%$ (41) of the fractures in this series.

Our findings confirm those of numerous studies showing that most fractures of the femur treated with titanium elastic nails heal well with few complications. Major complications were seen during the post-operative period in $17.5 \%$ of the fractures (41 of 234). Minor complications were seen in approximately $30 \%$ of the children, mostly associated with pain because of irritation by the nail. ${ }^{15}$ Anatomical alignment and equal leg-length were present in most of these children, suggesting that by careful attention to technique, such problems can be avoided. Most fractures $(65.8 \% ; 154$ of 234$)$ healed without complications.

The most common problem encountered was angulation exceeding the guidelines. ${ }^{16}$ This was seen in $18 \%$ of fractures of the distal third of the femur and in $9 \%$ of those in both the mid-shaft and proximal part.

Age was a strong predictor of outcome. The odds ratio for poor outcome was 3.86 for children aged 11 years and older compared with those who were below this age. Because remodelling is better in younger children, conventional guidelines recommend accepting greater amounts of angulation and shortening of the fracture if the child is less than ten years old. ${ }^{16}$ Consequently, the higher percentage of unsatisfactory results in children aged 11 years and older is due in part to the use of more stringent criteria for assessment. After adjusting for age, we could reach no conclusion regarding the association between location of the fracture, age, and loss of reduction. Because of the small sample size, no reliable conclusions can be drawn.

We found a significant difference between the weight of patients with poor as opposed to satisfactory or excellent outcomes. Since weight is associated with age, the association between weight and outcome was analysed separately. Outcomes could be ranked for 222 patients whose weights were recorded. The mean weight was $39 \mathrm{~kg}$ (17 to 95.2) for the group including both excellent and satisfactory outcomes, and $54 \mathrm{~kg}$ (22.3 to 95.2) for those with a poor outcome $(p=0.003)$. The maximum difference in the likelihood of a poor outcome was seen at a weight of $49 \mathrm{~kg}$; children weighing more than $49 \mathrm{~kg}$ were five times more likely to have a poor outcome than those weighing less than this.

Other clinical and radiological aspects of injury, fixation, and post-operative care did not predict outcome. The absence of a correlation between placement of the nail and a poor outcome is probably because of the adherence of the various surgeons to the guidelines for technique rather than an indication that the nail placement is of secondary importance.

One of the limitations of this study is the technique used for measuring flexion and extension. Because of the constraints of the radiographs available for retrospective review, flexion was measured from the shaft in most cases rather than by comparison with the normal bow of the opposite limb. Thus, flexion may have been slightly exaggerated. However, the sample size is large enough to reliably analyse differences in outcome, and, given the retrospective design of the study, some omissions in the data available for review must be accepted. The length of the follow-up available for each patient varied. Ideally the final outcome would be based on radiological and clinical data obtained once the patient had reached skeletal maturity, but it is difficult to arrange such an assessment. 
Titanium elastic nailing is currently the most popular method for treating fractures of the shaft of the femur in children of school age. Our results show that children older than 11 years and heavier than $49 \mathrm{~kg}$ are more likely to have a complication or a poor outcome. However, our study has confirmed that the outcome after treatment is excellent or satisfactory in most patients.

No benefits in any form have been received or will be received from a commercial party related directly or indirectly to the subject of this article.

\section{References}

1. Flynn JM, Luedtke LM, Ganley TJ. Comparison of titanium elastic nails with traction and a spica cast to treat femoral fractures in children. J Bone Joint Surg [Am] 2004;86-A:770-7.

2. Czertak DJ, Hennrikus WL. The treatment of pediatric femur fractures with early 90-90 spica casting. J Pediatr Orthop 1999;19:229-32.

3. Cases J, Gonzalez-Moran G, Albinana J. Femoral fractures in children from 4 years to 10 years: conservative treatment. J Pediatr Orthop B 2001;10:56-62.

4. Stans AA, Morrissy RT, Renwick SE. Femoral shaft fracture treatment in patients age 6-16 years. J Pediatr Orthop 1999:19:222-8.

5. Townsend DR, Hoffinger S. Intramedullary nailing of femoral shaft fractures in children via the trochanter tip. Clin Orthop 2000;376:113-18.

6. Letts M, Jarvis J, Lawton L, Davidson D. Complications of rigid intramedullary rodding of femoral shaft fractures in children. J Trauma 2002;52:504-16.

7. Ligier JN, Metaizeau JP, Prevot J, Lascombes P. Elastic stable intramedullary nailing of femoral shaft fractures in children. J Bone Joint Surg [Br] 1988;70-B:74-7.
8. Ligier JN, Metaizeau JP, Prevot J, Lascombes P. Elastic stable intramedullary pinning of long bone shaft fractures in children. Z Kinderchir 1985;40:209-12.

9. Prevot J, Lascombes P, Ligier JN. The ECMES (Centro-Medullary Elastic Stabilising Wiring) osteosynthesis method in limb fractures in children: principle, application on the femur: apropos of 250 fractures followed-up since 1979. Chirurgie 1993:119:473-6 (in French).

10. MehIman C, Foad S, Crawford A, Bhatt T. Weight $\geq 99$ pounds $(45 \mathrm{~kg})$ and age $\geq$ 12 years predict malunion following elastic stable intramedullary nailing (Nancy nailing) of femoral shaft fractures in children. Procs Pediatric Orthopaedic Society North America 2004 [abstract]

11. Flynn JM, Schwend RM. Management of pediatric femoral shaft fractures. J Am Acad Orthop Surg 2004;12:347-59.

12. Sanders J0, Brown RH, Mooney JF, et al. Treatment of femoral fractures in children by pediatric orthopedists: results of a 1998 survey. J Pediatr Orthop 2001;21:436-41.

13. Flynn JM, Skaggs DL, Sponseller PD, et al. The surgical management of pediatric fractures of the lower extremity. Instr Course Lect 2003;52:647-59.

14. Flynn JM, Hresko T, Reynolds RA, et al. Titanium elastic nails for pediatric femur fractures: a multicenter study of early results with analysis of complications. J Pediatr Orthop 2001:21:4-8.

15. Flynn JM, Luedtke L, Ganley TJ, Pill SG. Titanium elastic nails for pediatric femur fractures: lessons from the learning curve. Am J Orthop 2002:31:71-4.

16. Beaty JH, Kasser JR. (eds). Femoral shaft fractures. Sixth ed. In: Rockwood and Wilkins' fractures in children. Philadelphia: Lippincott, Williams \& Wilkins, 2001:893936

17. Bar-0n E, Sagiv S, Porat S. External fixation or flexible intramedullary nailing for femoral shaft fractures in children: a prospective, randomised study. J Bone Joint Surg [Br] 1997;79-B:975-8

18. Luhmann SJ, Schootman M, Schoenecker PL. Dobbs MB, Gordon JE. Complications of titanium elastic nails for pediatric femoral shaft fractures. J Pediatr Orthop 2003:23:443-7. 